Journal for ImmunoTherapy of Cancer

\title{
INfluenza Vaccine Indication During therapy with Immune checkpoint inhibitors: a multicenter prospective observational study (INVIDIa-2)
}

Melissa Bersanelli (D) , ${ }^{1,2}$ Diana Giannarelli, ${ }^{3}$ Ugo De Giorgi, ${ }^{4}$ Sandro Pignata, ${ }^{5}$ Massimo Di Maio, ${ }^{6,7}$ Alberto Clemente, ${ }^{8}$ Elena Verzoni, ${ }^{9}$ Raffaele Giusti, ${ }^{10}$ Marilena Di Napoli, ${ }^{5}$ Giuseppe Aprile, ${ }^{11}$ Paola Ermacora, ${ }^{12}$ Annamaria Catino, ${ }^{13}$ Vieri Scotti, ${ }^{14}$ Francesca Mazzoni, ${ }^{15}$ Pamela Francesca Guglielmini, ${ }^{16}$ Antonello Veccia, ${ }^{17}$ Marco Maruzzo, ${ }^{18}$ Ernesto Rossi, ${ }^{19}$ Francesco Grossi, ${ }^{20}$ Chiara Casadei, ${ }^{4}$ Corrado Ficorella, ${ }^{21}$ Vincenzo Montesarchio (D) ${ }^{22}$ Francesco Verderame, ${ }^{23}$ Mimma Rizzo, ${ }^{24}$ Giorgia Guaitoli, ${ }^{25}$ Lucia Fratino, ${ }^{26}$ Caterina Accettura, ${ }^{27}$ Manlio Mencoboni, ${ }^{28}$ Fable Zustovich, ${ }^{29}$ Cinzia Baldessari, ${ }^{25}$ Saverio Cinieri, ${ }^{30}$ Andrea Camerini, ${ }^{31}$ Letizia Laera, ${ }^{32}$ Mariella Sorarù, ${ }^{33}$ Paolo Andrea Zucali, ${ }^{34,35}$ Valentina Guadalupi, ${ }^{36}$ Francesco Leonardi, $^{2}$ Marcello Tiseo, ${ }^{1,2}$ Michele Tognetto, ${ }^{2}$ Francesco Di Costanzo, ${ }^{37}$ Carmine Pinto, ${ }^{38,39}$ Giorgia Negrini, ${ }^{40}$ Antonio Russo, ${ }^{41}$ Maria R Migliorino, ${ }^{42}$ Marco Filetti, ${ }^{43}$ Sebastiano Buti (D) , ${ }^{2}$ FICOG group (Federation of Italian Cooperative Oncology Groups)

To cite: Bersanelli M, Giannarelli D, De Giorgi U, et al. INfluenza Vaccine Indication During therapy with Immune checkpoint inhibitors: a multicenter prospective observational study (INVIDla-2). Journal for ImmunoTherapy of Cancer 2021;9:e002619. doi:10.1136/jitc-2021-002619

- Additional material is published online only. To view, please visit the journal online (http://dx.doi.org/10.1136/jitc2021-002619).

Accepted 23 March 2021

Check for updates

(C) Author(s) (or their employer(s)) 2021. Re-use permitted under CC BY-NC. No commercial re-use. See rights and permissions. Published by BMJ.

For numbered affiliations see end of article.

Correspondence to Dr Melissa Bersanelli; bersamel@libero.it

\section{ABSTRACT}

Background Until now, no robust data supported the efficacy, safety and recommendation for influenza vaccination in patients with cancer receiving immune checkpoint inhibitors (ICls).

Methods The prospective multicenter observational INfluenza Vaccine Indication During therapy with Immune checkpoint inhibitors (INVIDla-2) study investigated the clinical effectiveness of influenza vaccination in patients with advanced cancer receiving ICls, enrolled in 82 Italian centers from October 2019 to January 2020. The primary endpoint was the time-adjusted incidence of influenza-

like illness (ILI) until April 30, 2020. Secondary endpoints regarded ILI severity and vaccine safety.

Results The study enrolled 1279 patients; 1188 patients were evaluable for the primary endpoint analysis. of them, $48.9 \%$ (581) received influenza vaccination. The overall ILI incidence was $8.2 \%$ (98 patients). Vaccinated patients were significantly more frequently elderly $(p<0.0001)$, males $(p=0.004)$, with poor European Cooperative Oncology Group performance status $(p=0.009)$, affected by lung cancer $(p=0.01)$, and by other non-cancer comorbidities $(p<0.0001)$ when compared with unvaccinated. ILI incidence was not different basing on influenza vaccination: the time-to-ILI was similar in vaccinated and unvaccinated patients $(p=0.62)$. ILI complications were significantly less frequent for patients receiving the vaccination $(11.8 \%$ vs $38.3 \%$ in unvaccinated, $p=0.002$ ). ILI-related intravenous therapies were significantly less frequent in vaccinated patients than in unvaccinated $(11.8 \%$ vs $29.8 \%, \mathrm{p}=0.027)$. ILI lethality was, respectively, $0 \%$ in vaccinated and $4.3 \%$ in unvaccinated patients. Vaccine-related adverse events were rare and mild (1.5\%, grades $1-2)$.

Conclusion The INVIDla-2 study results support a positive recommendation for influenza vaccination in patients with advanced cancer receiving immunotherapy.

\section{INTRODUCTION}

Seasonal influenza is an acute respiratory infection caused by influenza viruses circulating worldwide. Type A and type B influenza viruses cause seasonal disease epidemics, mainly during winter. ${ }^{1}$ According to recent estimates, 4.0-8.8 influenza-associated respiratory deaths per 100000 individuals occur annually all over the world. The highest mortality rates were estimated among people aged 75 years or older (51.3-99.4 per 100000 individuals). ${ }^{2}$ The diagnosis of influenza is mainly clinical, given the laboratory tests' low predictive capacity. ${ }^{3}$ Influenza-like illness (ILI) was defined to guarantee the greatest possible homogeneity in detection by the European Centre for Disease Prevention and Control (ECDC) in 2008 and updated in 2018 , as the sudden and rapid onset of one or more of both general and respiratory symptoms. $^{34}$

Influenza infection is a potential cause of additional morbidity and mortality in 
immunocompromised individuals, such as patients with cancer, because of the underlying disease or its immunosuppressive therapy. ${ }^{5}$ Patients with advanced cancer receiving chemotherapy are at high risk of severe events related to influenza complications, and case-fatality rates of about $10 \%$ were reported by the literature. ${ }^{56}$ In addition, influenza complications can lead to the interruption or delay of the anticancer therapy, and concomitant drugs (such as antibiotics) used to treat bacterial superinfections can negatively impact its efficacy. ${ }^{78}$

Vaccination is the most efficacious form to prevent influenza and ILI-related lethality. Despite low seroconversion rates demonstrated in patients receiving cytotoxic therapy, the clinical benefit seems to overweight the potential risks when vaccinating adults with cancer against influenza. ${ }^{9}$ Oncological guidelines recommend the vaccine in patients with advanced cancer undergoing active treatment: nevertheless, this indication is often disregarded, especially in the case of non-cytotoxic therapies. ${ }^{11} 12$

On the other hand, the susceptibility of patients with advanced cancer treated with new-generation immunotherapy based on immune checkpoint inhibitors (ICIs) to viral infections has not been investigated. In recent years, the introduction of ICIs in the clinical scenario has constituted a profound innovation in advanced solid tumors' systemic treatment. The aim of the new immunotherapy is restoring cellular immunocompetence against the tumor: it is, therefore, plausible that individuals treated with ICIs may be more immunocompetent, when compared with those treated with chemotherapy, also against viral infections. Along this line, more potent immune stimulation after vaccines were described in these patients, even when compared with healthy individuals. ${ }^{13} 14$

Until now, no robust data supported the need, efficacy and safety of influenza vaccination in patients with cancer receiving ICI immunotherapy. The current evidence is limited, mainly retrospective and controversial, ${ }^{15-18}$ with no prospective trials supporting influenza vaccine recommendation and management in this cancer patient subgroup.

Considering the unmet need for the counseling of patients with advanced cancer receiving ICI-based therapy concerning influenza vaccination, we designed a multicenter prospective observational study to investigate the impact of vaccination on incidence, severity, and lethality of ILI in this population.

\section{METHODS}

\section{INVIDla-2 study design and participants}

The INfluenza Vaccine Indication During therapy with Immune checkpoint inhibitors (INVIDIa-2) study was a multicenter prospective observational trial. The primary objective was to investigate the effectiveness of influenza vaccine administration in terms of incidence and severity of ILI in patients with advanced cancer undergoing systemic treatment with ICIs. The secondary objectives of the study regarded influenza vaccine safety during immunotherapy and the oncological outcome of patients to ICIs basing on the vaccine administration or influenza occurrence.

All patients with advanced solid tumors receiving therapy with ICI (alone or in combinations) from October 1, 2019, to January 31, 2020 (corresponding to the influenza vaccinal season) were eligible for enrollment. ILI, according to ECDC, was defined as a sudden and rapid onset of one or more of the following general symptoms: fever or low-grade fever, malaise and/or fatigue, headache, myalgia; and at least one of the following respiratory symptoms: cough, sore throat, dyspnea. "Sudden and rapid" is taken to mean to the extent that the date of symptom onset can be clearly identified. ${ }^{34}$

Since placebo-controlled randomized controlled trials of antiviral vaccination among adults with cancer are often considered ethically questionable ${ }^{19}$ and foreseeing a proportional numeric distribution due to the relatively low vaccine coverage expected in this population, ${ }^{12}$ we opted for an observational approach. We prespecified a comparative analysis of clinical characteristics between the two groups of vaccinated and unvaccinated patients to measure the indication bias deriving from the study's observational nature.

The study's primary endpoint was the time-adjusted ILI incidence, calculated in terms of time-to-ILI (TTI), from October 1, 2020, until April 30, 2020. Such a timeadjusted endpoint was chosen to weight the rate of ILI occurrence based on the time exposure of patients to the influenza season, considering the possibility of late enrollment or early death during the predefined period of the ILI observation. TTI was defined as the time interval between the date of enrollment, and the date of ILI symptoms occurrence; patients not experiencing ILI at the cut-off date of April 30, 2020, were considered as censored at this date or the date of death if previously occurred.

The secondary endpoints regarding ILI severity were the incidence of ILI-related complications, defined as pneumonia confirmed by imaging, laboratory-confirmed bacterial superinfections, or other severe complications; the ILI-related intravenous therapy administration; the ILI-related hospitalization rate; the duration of ILI symptoms (in days), the ILI lethality. Vaccination safety was recorded as a secondary endpoint in terms of vaccinerelated adverse events (VRAEs), defined by the suspicion of a causal relationship between the influenza vaccine and the adverse reaction, and classified by Common Terminology Criteria for Adverse Events (CTCAE) V.5.0. ${ }^{20}$

Secondary endpoints regarding the patients' oncological outcome to ICI treatment will also be measured in future analysis in terms of ICI safety, activity and efficacy (follow-up still ongoing as per protocol).

The influenza season, from October 2019 to April 2020, constituted the ILI observation period for both the primary and secondary endpoints regarding the severity of ILI. The timing of the study is shown in figure 1A. 
A

INVIDla-2 study duration 16 months

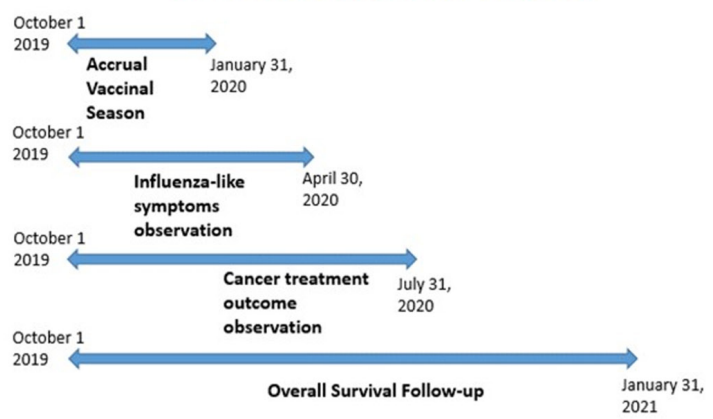

B

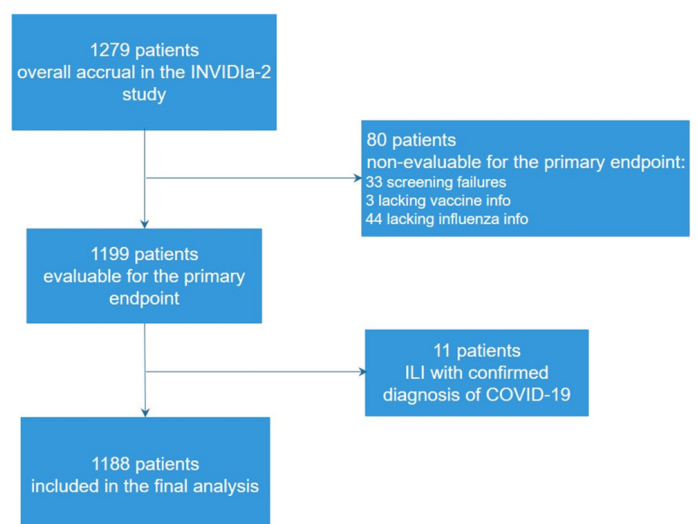

Figure 1 Patient disposition in the INfluenza Vaccine Indication During therapy with Immune checkpoint inhibitors (INVIDla-2) study.

Prespecified subgroup analyses were planned for elderly and patients with lung cancer, as populations at high-risk of ILI-related complications. Two prespecified age cutoffs were used for operational purpose: 65 years, which is the age to recommend influenza vaccination in Italy, and the median age of the study population, to consider equal size subgroups. The type of influenza vaccine received, the vaccination date, the figure recommending vaccination, and information about any other vaccine administered after the study enrollment were recorded, together with clinical characteristics and outcomes of patients about ILI, other viral or bacterial infections, and cancer disease and therapy. The study data were prospectively collected in a validated eCRF platform.

The study's funders had no role in study design, data collection, data analysis, data interpretation, or writing of the report. The corresponding author had full access to all the data in the study and had final responsibility for the publication.

\section{Sample size and statistical analysis}

Based on an expected ILI incidence of $12 \%$ for unvaccinated ICI-treated patients with cancer, ${ }^{15}$ establishing a $50 \%$ reduction in the incidence of ILI in vaccinated patients as the reliable objective of the vaccination, statistical power of $80 \%$ and statistical significance with $\mathrm{p}<0.05$, and hypothesizing vaccine coverage of $75 \%$ (based on WHO recommendation for categories at high risk $^{21}$ ), an overall study sample of 974 patients was calculated. An overaccrual of $30 \%$ was preplanned based on the expected rate of screening failures, considering the trial's observational nature. The study involved 82 Italian centers.

Data related to quantitative variables were reported using median and IQR and absolute count and percentages when referring to categorical items. The exact $95 \%$ CIs were calculated for proportions. Association between categorical variables was evaluated with the $\chi^{2}$ test. TTI curves were estimated by the Kaplan-Meier method and compared with the log-rank test. The Cox proportional hazard model was used to assess associations between demographic and clinical factors and derive HRs and 95\% CI. A logistic regression model was used when evaluating ILI complications, and OR with their 95\% CI were reported. All the statistical tests were two tailed.

\section{RESULTS \\ Patient disposition}

The INVIDIa-2 study prospectively enrolled 1279 patients with advanced cancer receiving ICIs. Of them, 1188 were eligible for the primary analysis (patient disposition is showed in figure 1B). The characteristics of the study population are summarized in table 1 .

Overall, 581 patients received influenza vaccination (48.9\%) while 607 did not (51.1\%). Vaccinated patients were significantly more frequently elderly $(p<0.0001)$, males $(\mathrm{p}=0.004)$, with poor European Cooperative Oncology Group (ECOG) performance status (PS, $\mathrm{p}=0.009)$, affected by lung cancer $(\mathrm{p}=0.01)$, and by other non-cancer comorbidities $(p<0.0001)$ when compared with unvaccinated (table 1). Indication for influenza vaccination was given by general practitioners in $64.1 \%$ of cases, clinical oncologists in $16.0 \%$, unsolicited patients' decision in $12.5 \%$, other physicians in $7.4 \%$. The vaccine types administered in the study population are showed in online supplemental table S1. Patients received other vaccines during the study conduct: 45 received pneumococcal vaccination (43 of whom together with influenza vaccine), two patients received tetanus vaccination.

\section{Incidence and severity of ILI}

The incidence of ILI in the overall study population was 98 cases $(8.2 \%, 95 \%$ CI 6.7 to 10.0$)$. The ILI clinical manifestation in these patients, considering the ECDC definition, is represented in figure 2. The median duration of ILI symptoms was 7 days (IQR 4-14). The overall complication rate was $24.5 \%$, with pneumonia developed during ILI in 21 cases $(21.4 \%)$ and other complications in $3(3.1 \%)$; intravenous therapies due to ILI were administered in $20.4 \%$ of all ILI cases, and ILI-related hospital admission was required in $11.2 \%$ of cases. 
Table 1 Characteristics of patients in the overall study population and comparative analysis of patient characteristics basing on vaccine subgroups

\begin{tabular}{|c|c|c|c|c|}
\hline & $\begin{array}{l}\text { Overall population } \\
\mathrm{n}=1188\end{array}$ & $\begin{array}{l}\text { Vaccinated } \\
\mathrm{n}=581\end{array}$ & $\begin{array}{l}\text { Unvaccinated } \\
\mathrm{n}=607\end{array}$ & $P$ value \\
\hline AGE (median, IQR, range) & 69 (61-76), (20-93) & 72 (64-77), (30-90) & 66 (58-73), (20-93) & $<0.0001$ \\
\hline \multicolumn{5}{|l|}{ Gender } \\
\hline M & 831 (69.9) & $429(73.8)$ & $402(66.2)$ & 0.004 \\
\hline $\mathrm{F}$ & $357(30.1)$ & $152(26.2)$ & $205(33.8)$ & \\
\hline ECOG PS & & & & (0 vs $1-2-3$ ) \\
\hline 0 & $686(57.7)$ & $312(53.7)$ & $374(61.6)$ & 0.009 \\
\hline 1 & 431 (36.3) & $235(40.4)$ & 196 (32.3) & \\
\hline 2 & $53(4.5)$ & $22(3.8)$ & $31(5.1)$ & \\
\hline 3 & $3(0.3)$ & $2(0.3)$ & $1(0.2)$ & \\
\hline Unknown & $15(1.3)$ & $10(1.7)$ & $5(0.8)$ & \\
\hline \multicolumn{5}{|l|}{ Primary tumor } \\
\hline Lung & 645 (54.3) & $337(58.0)$ & $308(50.7)$ & (lung vs all others) \\
\hline $\mathrm{RCC}$ & 201 (16.9) & $107(18.4)$ & $94(15.5)$ & 0.01 \\
\hline Melanoma & $153(12.9)$ & $52(9.0)$ & $101(16.6)$ & (RCC vs all others) \\
\hline UC & $64(5.4)$ & $29(5.0)$ & $35(5.8)$ & 0.18 \\
\hline $\mathrm{H} \& \mathrm{~N}$ & $41(3.5)$ & $14(2.4)$ & $27(4.4)$ & (melanoma vs all others) \\
\hline Other & $84(7.1)$ & $42(7.2)$ & $42(6.9)$ & 0.0001 \\
\hline $\mathrm{ICI}$ treatment line & & & & 0.69 \\
\hline 1 & $663(55.8)$ & $316(54.4)$ & 347 (57.2) & \\
\hline 2 & $426(35.9)$ & $214(36.8)$ & $212(34.9)$ & \\
\hline 3 & $96(8.0)$ & $48(8.3)$ & $48(7.9)$ & \\
\hline Unknown & $3(0.3)$ & $3(0.5)$ & 0 & \\
\hline Splenectomy & & & & 0.38 \\
\hline Yes & $8(0.7)$ & $5(0.9)$ & $3(0.5)$ & \\
\hline No & $915(77.0)$ & $455(78.3)$ & $460(75.8)$ & \\
\hline Unknown & $265(22.3)$ & $121(20.8)$ & $144(23.7)$ & \\
\hline Therapy & & & & 0.80 \\
\hline $\mathrm{ICl} / \mathrm{ICl}+\mathrm{ICl}$ & $1075(90.5)$ & $527(90.7)$ & $548(90.3)$ & \\
\hline $\mathrm{ICl}+$ Other $^{*}$ & $113(9.5)$ & $54(9.3)$ & $59(9.7)$ & \\
\hline $\begin{array}{l}\text { Immunotherapy } \\
\text { type }\end{array}$ & & & & 0.50 \\
\hline Single agent (ICl) & $1122(94.4)$ & $546(94.0)$ & $576(94.9)$ & \\
\hline Combinations $(\mathrm{ICl}+\mathrm{ICl})$ & $66(5.6)$ & $35(6.0)$ & $31(5.1)$ & \\
\hline Comorbidity & & & & $<0.0001$ \\
\hline Yes & $875(73.7)$ & 467 (86.4) & $408(67.2)$ & \\
\hline No & $313(26.3)$ & $114(19.6)$ & $199(32.8)$ & \\
\hline \multicolumn{5}{|l|}{ Comorbidity type } \\
\hline Cardiovascular & $202(17.0)$ & $118(20.3)$ & $84(13.8)$ & 0.003 \\
\hline Asthma/COPD & $401(33.8)$ & $225(38.7)$ & $176(29.0)$ & $<0.0001$ \\
\hline Diabetes & $185(15.6)$ & $98(16.9)$ & $87(14.3)$ & 0.23 \\
\hline Others & $283(23.8)$ & $143(24.6)$ & $140(23.1)$ & 0.53 \\
\hline Smoking habits & & & & $<0.0001$ \\
\hline Current & 287 (24.2) & $125(21.5)$ & 207 (34.1) & \\
\hline Former & $512(43.1)$ & $288(49.6)$ & 224 (36.9) & \\
\hline
\end{tabular}


Table 1 Continued

\begin{tabular}{lcccc}
\hline & $\begin{array}{l}\text { Overall population } \\
\mathrm{n}=1188\end{array}$ & $\begin{array}{l}\text { Vaccinated } \\
\mathbf{n = 5 8 1}\end{array}$ & $\begin{array}{l}\text { Unvaccinated } \\
\mathbf{n = 6 0 7}\end{array}$ & P value \\
\hline Never & $358(30.1)$ & $151(26.0)$ & $162(26.7)$ \\
Unknown & $31(2.6)$ & $17(2.9)$ & $14(2.3)$ \\
\hline
\end{tabular}

COPD, chronic obstructive pulmonary disease; H\&N, head and neck carcinoma; ICI, immune checkpoint inhibitor; ECOG PS, European Cooperative Oncology Group Performance Status; RCC, renal cell carcinoma; UC, urothelial cancer.

The time-adjusted ILI incidence, in terms of TTI, was similar in vaccinated and unvaccinated patients (figure 3, $\mathrm{p}=0.62$ ). The crude incidence of ILI was $8.8 \%$ (95\% CI 6.6 to 11.4$)$ in vaccinated patients $(51 / 581)$ versus $7.7 \%-$ $95 \%$ CI 5.7 to 10.2 in unvaccinated patients $(47 / 607)$ $(\mathrm{p}=0.52)$. Also, the median duration of ILI symptoms was similar in vaccinated and unvaccinated subjects (7 days and 8.5 days, respectively, $\mathrm{p}=0.82$ ).

ILI complications were significantly less frequent for patients receiving vaccination than for unvaccinated patients: respectively, the complication rate was $11.8 \%$ $(6 / 51)$ versus $38.3 \%$ (18/47), $\mathrm{p}=0.002$ (figure 4$)$. The type of complications found in the two groups is summarized in online supplemental table S2. ILI-related intravenous therapies were also significantly less frequent in vaccinated patients than in unvaccinated: $11.8 \%$ versus $29.8 \%(\mathrm{p}=0.027)$.

Hospital admission due to ILI occurred for 11 patients (11.2\% of all ILI cases); of them, 7 were unvaccinated (ILIrelated hospitalization was required for $7 / 47$ (14.9\%) of unvaccinated versus $4 / 51$ (7.8\%) of vaccinated patients, $\mathrm{p}=0.27$ ).

ILI lethality was $2 \%$ overall (2/98 patients, both unvaccinated). Within vaccine groups, ILI lethality was $0 / 51$ $(0 \%)$ in vaccinated versus $2 / 47(4.3 \%)$ in unvaccinated patients.

Considering the respective timings of influenza vaccination and ILI occurrence, 4 patients $(4.1 \%)$ developed ILI within 2 weeks after their vaccination, and $5(5.1 \%)$ had the vaccine after a prior episode of ILI. Overall, these nine patients were likely not protected by their vaccination: nevertheless, the study results remained unchanged when removing these cases from the analyses (data not shown).

The relative vaccine effectiveness of adjuvanted versus non-adjuvanted vaccines in preventing ILI was 54.9\% (6.3\% vs $9.7 \%)$; this difference however was not statistically significant $(\mathrm{p}=0.21)$.

\section{Multivariate analyses}

A Cox regression model was implemented to evaluate associations between demographic and clinical characteristics and TTI. The following were considered: sex, age, ECOG-PS, comorbidities (respiratory, cardiovascular or metabolic comorbidity), lung cancer as the primary tumor, influenza vaccination. After a selection based on $p$ values, lung cancer $(p=0.009)$, respiratory comorbidities $(\mathrm{p}=0.007)$ and age $<69$ years $(\mathrm{p}=0.028)$ resulted as independent factors associated with higher ILI incidence (table 2). When considering only vaccinated patients $(n=581)$, none of the considered factors were associated with ILI incidence (online supplemental table S3).

When considering patients developing ILI $(n=98)$, the only factor inversely associated with ILI-related complications at the multivariate analysis was the vaccine administration $(\mathrm{p}=0.004$, table 2$)$.

\section{Influenza vaccine safety}

Overall, 9 of 581 patients had VRAEs: local erythema, local pain, local edema, fever, heart-pounding, rash and pruritus were reported. The overall incidence of VRAEs was $1.5 \%$, with grade $(\mathrm{G}) 1-2$ according to CTCAE 5.0, self-limiting course and complete resolution; no G3-4 VRAEs were reported.

\section{Subgroups of interest: elderly and patients with lung cancer}

Overall, elderly patients ( $\geq 69$ years) had non-statistically significantly longer TTI $(\mathrm{p}=0.15)$ than non-elderly. Patients affected by lung cancer had statistically significantly shorter TTI than patients with other primary tumors (online supplemental figure S4). The crude incidence of ILI in patients with lung cancer was also higher, $10.1 \%$ vs $6.1 \%$ in patients with non-lung tumors, respectively ( $\mathrm{p}=0.013)$.

Considering the impact of influenza vaccination in these subgroups, it was consistent with that on the overall population: ILI incidence was similar irrespective of vaccination (online supplemental figure S5), and the rates of complications were lower in vaccinated patients when compared with those of unvaccinated individuals (online supplemental table S6).

\section{DISCUSSION}

The INVIDIa-2 study is the first large prospective trial investigating influenza vaccination's clinical effectiveness in patients with cancer treated with immunotherapy. The target population, constituted by individuals undergoing immune checkpoint blockade, represents a growing reality in oncology. The implications of the new immunological framework characterizing these patients are still largely unexplored, often generating doubts about the management of common indications, such as in vaccine counseling.

In the present study, in patients with advanced cancer treated with immunotherapy, ILI incidence was not 


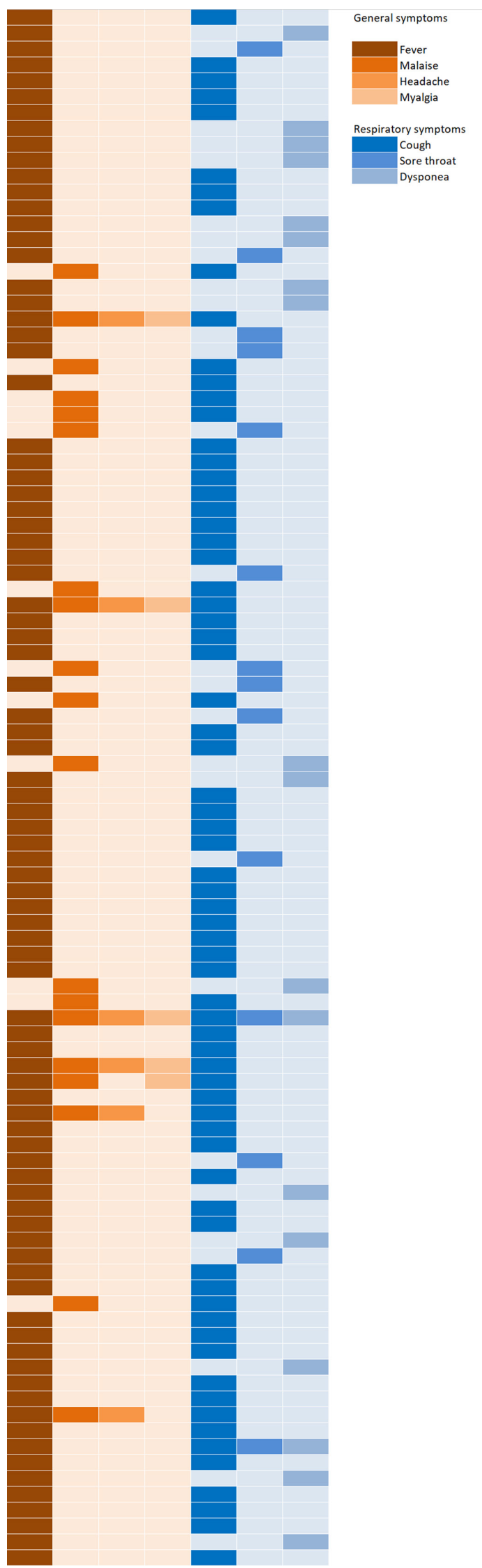

Figure 2 Clinical heatmap representing the pattern of symptoms in patients developing influenza-like illness (each row represents a single patient) according to the European Centre for Disease Prevention and Control definition.

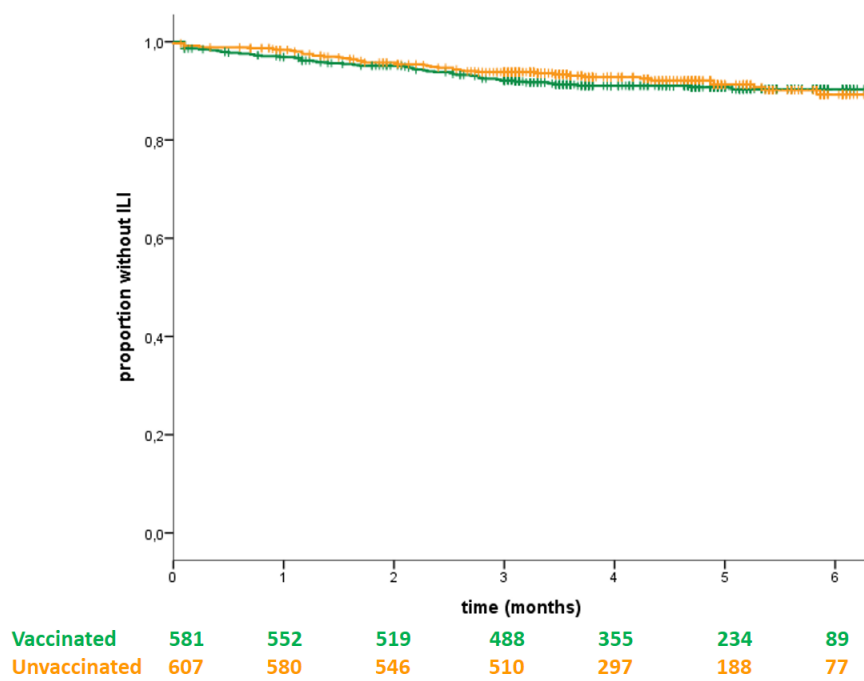

Figure 3 Kaplan-Meier curve representing the time to influenza-like-illness (ILI) in vaccinated and unvaccinated patients.

different according to influenza vaccination. Nevertheless, despite an evident unfavorable clinical profile of the vaccinated group (at high risk of severe complications due to older age, poorer ECOG PS, lung cancer frequency, and respiratory and cardiovascular comorbidities) influenza vaccine administration was related to a significantly lower rate of ILI complications (and to the reduced need of intravenous therapy) in comparison with the unvaccinated group ( $11.8 \%$ vs $38.3 \%, \mathrm{p}=0.002$ ). The protective effect of vaccination in terms of ILI complications was confirmed at the multivariate analysis (Table 2).

Of note, the lack of randomization and the consequent negative selection of vaccinated patients with high-risk characteristics for ILI occurrence (ie, lung cancer prevalence) could have masked the vaccine's efficacy in terms of ILI incidence. The results of the multivariate analyses are in support of this hypothesis, as lung cancer and respiratory comorbidities were associated with a higher incidence of ILI (table 2), but the impact of such variables was canceled when considering the vaccinated group only (online supplemental table S3).

In further support of the vaccine, hospitalization due to ILI was infrequent in vaccinated patients, and no ILIrelated deaths were reported in patients receiving the vaccination, while two ILI-related deaths occurred in unvaccinated patients. Despite the lack of statistical significance due to relatively small numbers, such findings are clinically meaningful, especially compared with previous lethality data in patients treated with chemotherapy. ${ }^{56}$ From this standpoint, the INVIDIa-2 study highlighted the peculiarity of immunological status of patients with cancer receiving immunotherapy. The study finally allows basing these patients' counseling about influenza vaccination not on extrapolation but on evidence specifically provided in ICI-treated patients with cancer.

In previous studies, both serological and cell-mediated responses to vaccines were more pronounced in this 


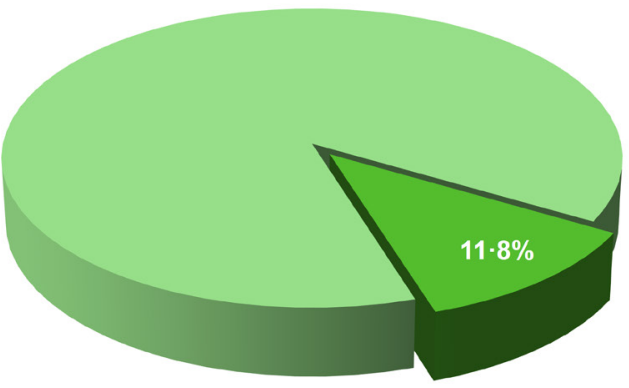

- Complicated ILI

Non-complicated ILI

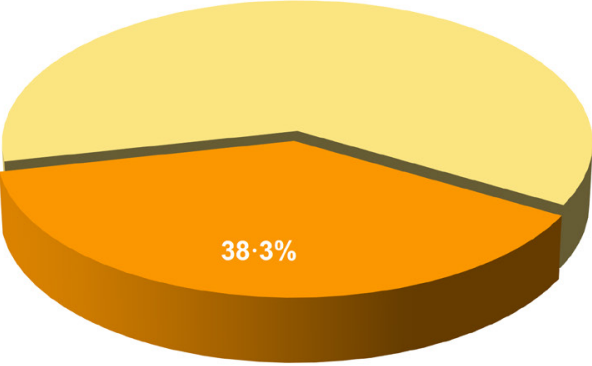

- Complicated ILI Non-complicated ILI

Figure 4 Pie charts representing the rates of influenza-like illness (ILI)-related complications in vaccinated and unvaccinated patients.

population. Flow cytometric analyses recently revealed that influenza vaccination elicited higher influenza-specific cell-mediated immune responses in patients receiving ICIs than those receiving cytotoxic chemotherapy. ${ }^{22}$ Similarly, if the reported seroconversion rate of patients with cancer undergoing chemotherapy ranged from $8 \%$ to $28 \%,{ }^{23}$ that reported for patients with cancer undergoing immune checkpoint blockade was higher, ranging from
$60 \%$ to $70 \% .^{2425}$ The limitations of such analyses were the small sample size and the lack of correlation with the clinical efficacy of vaccination, which was not investigated in these case series. To address the issue, an ancillary analysis of the INVIDIa-2 trial (INVIDIa-bios substudy ${ }^{26}$ ) allowed to collect serial serum samples and peripheral blood mononuclear cells samples of 130 subjects enrolled. The analyses are currently ongoing to confirm the previously

Table 2 Multivariate analysis based on time to influenza-like-illness (TTI) in the overall study population (a, Cox regression analysis) and complication rate in the subgroup of patients developing influenza-like illness (ILI) (b, logistic regression analysis)

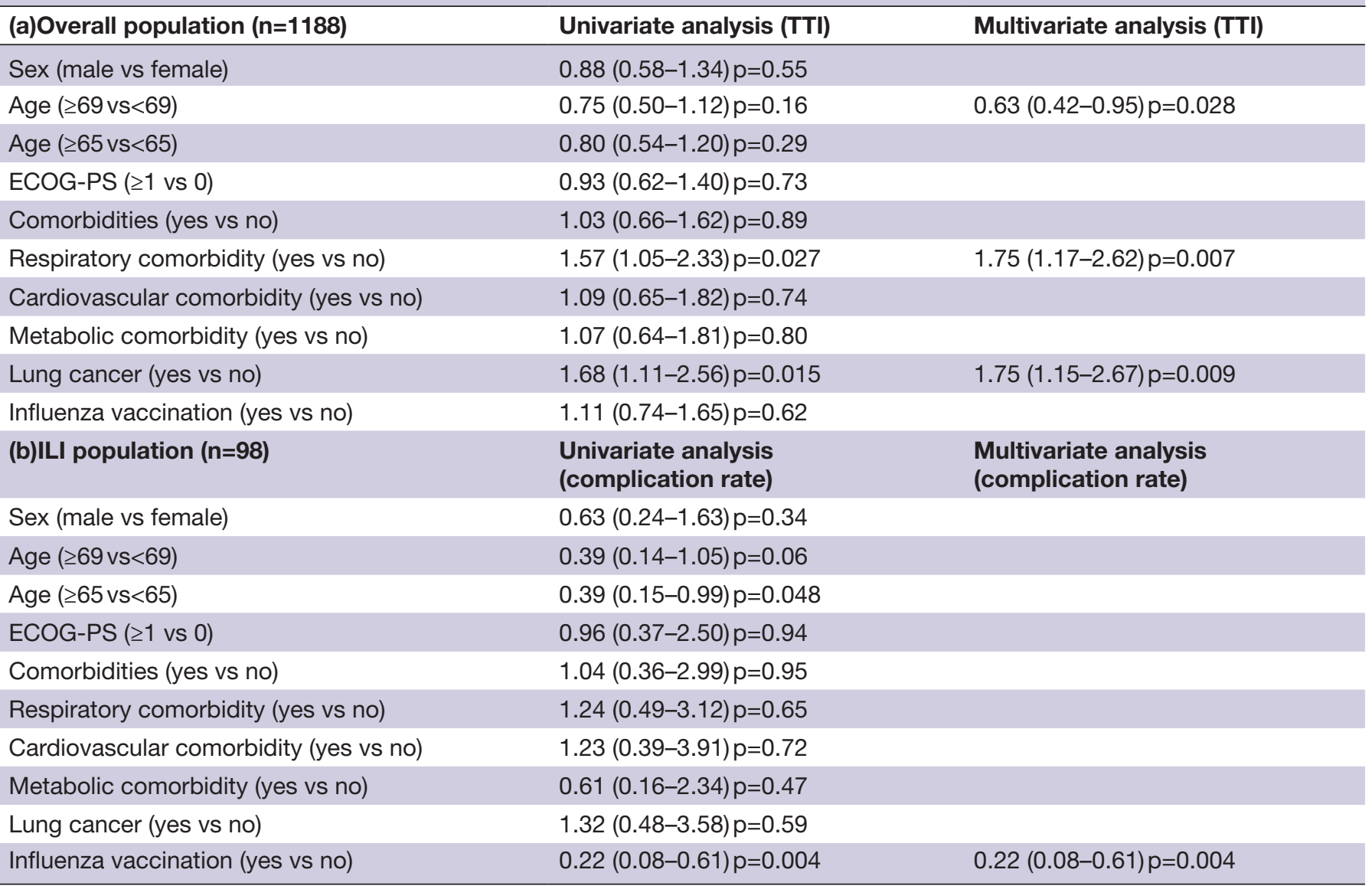

ECOG PS, European Cooperative Oncology Group Performance Status; ILI, influenzalike illness; TTI, time to influenza-like illness. 
cited evidence in this larger prospective population, then matching the laboratory results with clinical outcomes in terms of ILI incidence, severity, and lethality.

After the prior evidence in favor of serological activity of influenza vaccine in patients with cancer receiving ICIs, the present study constitutes the first prospective demonstration of its clinical effectiveness. Irrespective of the possible inaccuracy of the clinical diagnosis of influenza based on ILI, it is likely that the ILI complications are more reliable than the ILI incidence, realistically marking the actual influenza episodes in this population. As a matter of fact, the other various respiratory infections usually circulating during the influenza season (such as rhinovirus and adenovirus) are not frequently responsible for severe complications, while 30\%-40\% of hospitalized patients with laboratory-confirmed influenza are diagnosed with acute pneumonia, and bacterial superinfections are described in $30 \%-50 \%$ of cases. ${ }^{27}$ Influenza A (H1N1) virus alone was found responsible for more than $50 \%$ of severe acute respiratory infection cases leading to hospitalization in a large series of patients. ${ }^{28} \mathrm{Of}$ note, the patients enrolled in the INVIDIa-2 study developing ILI with the diagnosis of COVID-19 were excluded from the present analyses: since the first isolation of the SARS-CoV-2 virus in our Country in February 2019, all patients with cancer developing ILI symptoms were systematically tested for COVID-19, and the data about swabs were prospectively recorded in the study platform. The results about patients with COVID-19 ILI enrolled in the INVIDIa-2 study have been preliminary reported. ${ }^{29}$ Moreover, pneumonitis related to ICI or local therapies (eg, radiotherapy) or tumor progression was also separately recorded, allowing the proper identification of ILIrelated pneumonia.

Another relevant message of the study, to be immediately translated in clinical practice, is that all patient subgroups benefited from influenza vaccination, reducing ILI-related complications, irrespectively of age and primary tumor. The common tendency of recommending the vaccine only for patients aged over-65 or those with respiratory impairment due to primary lung cancer should be replaced by a general positive recommendation, supported by meaningful benefit also in younger patients and other subgroups.

Finally, vaccine safety's critical issue seems to reassure the oncologists about the low rate of VRAEs, mostly mild and self-limiting, reported in the INVIDIa-2 study population. Of note, the analysis of immune-related adverse events to ICI immunotherapy is ongoing, together with the oncological outcome, with longer follow-up.

Beyond the ethically unavoidable observational nature, the present study's limitation is represented by the lack of statistical power to properly analyze different vaccine subgroups, preventing reliable conclusions about the vaccine strategy. The possible higher efficacy of adjuvanted vaccines should be furtherly investigated in the ICI-treated cancer patient population, once again separately from chemotherapy-treated subjects.
Regarding the apparent lack of benefit in terms of ILI incidence, even if the expected efficacy of influenza vaccination remains relatively low in patients with cancer, epidemiological estimates by the United States Centers for Disease Control and Prevention demonstrated that the more people vaccinated in a population, the larger effect the vaccine has even when vaccine effectiveness is low. ${ }^{30}$ Along this line, the promotion of influenza vaccination is even more important during the pandemic spread of SARS-CoV-2, given the current threat of concurrent influenza and COVID-19 epidemics. ${ }^{31}$

In conclusion, the INVIDIa-2 study results are immediately transposable into clinical practice, supporting influenza vaccination recommendation in patients with advanced cancer receiving ICI immunotherapy.

\section{Author affiliations}

${ }^{1}$ Medicine and Surgery Department, University of Parma, Parma, Italy

${ }^{2}$ Medical Oncology Unit, University Hospital of Parma, Parma, Italy

${ }^{3}$ Biostatistical Unit, Regina Elena National Cancer Institute, IRCCS, Rome, Italy

${ }^{4}$ Department of Medical Oncology, Istituto Scientifico Romagnolo per lo Studio e la Cura dei Tumori (IRST) IRCCS, Meldola, Italy

${ }^{5}$ UC Oncologia Medica Uro-Ginecologica, Istituto Nazionale Tumori "Fondazione G.

Pascale", IRCCS, Napoli, Italy

${ }^{6}$ Department of Oncology, University of Turin, Torino, Italy

${ }^{7}$ Medical Oncology, Azienda Ospedaliera Ordine Mauriziano di Torino, Torino, Italy

${ }^{8}$ Biostatistics and Clinical Research Unit, Istituto Scientifico Romagnolo per lo Studio e la Cura dei Tumori (IRST) IRCCS, Meldola, Italy

${ }^{9}$ SS.Oncologia Genitourinaria, Fondazione IRCCS Istituto Nazionale dei Tumori,

Milano, Italy

${ }^{10}$ Medical Oncology Unit, Sant'Andrea Hospital, Roma, Italy

${ }^{11}$ Department of Oncology, San Bortolo General Hospital, Vicenza, Italy

${ }^{12}$ Dipartimento di Oncologia, Presidio Ospedaliero Universitario Santa Maria della Misericordia, Azienda sanitaria universitaria integrata Friuli Centrale, Udine, Italy

${ }^{13}$ Medical Thoracic Oncology Unit, IRCCS Istituto Tumori "Giovanni Paolo II", Bari, Italy

${ }^{14}$ Radiation Oncology Unit, Azienda Ospedaliero-Universitaria Careggi, Firenze, Italy

${ }^{15}$ Medical Oncology Unit, University Hospital Careggi, Firenze, Italy

${ }^{16}$ SC Oncologia, AO SS Antonio e Biagio e C. Arrigo, Alessandria, Italy

${ }^{17}$ Medical Oncology, Santa Chiara Hospital, Trento, Italy

${ }^{18}$ Oncologia Medica 1, Istituto Oncologico Veneto IOV-IRCCS, Padua, Italy

${ }^{19}$ Medical Oncology, Fondazione Policlinico Universitario A. Gemelli IRCCS, Rome, Italy

${ }^{20}$ Medical Oncology Department, Fondazione IRCCS Ca' Granda Ospedale Maggiore Policlinico, Milan, Italy

${ }^{21}$ Department of Biotechnological and Applied Clinical Sciences, St Salvatore Hospital, University of L'Aquila, L'Aquila, Italy

${ }^{22}$ UOC Oncologia, Azienda Ospedaliera Specialistica dei Colli, Ospedale Monaldi, Napoli, Italy

${ }^{23}$ Oncology Department, Ospedali Riuniti Villa Sofia Cervello, Palermo, Italy

${ }^{24}$ Oncologia Traslazionale, Istituti Clinici Scientifici Maugeri IRCCS, Pavia, Italy

${ }^{25}$ Medical Oncology Unit, Universita degli Studi di Modena e Reggio Emilia, Modena, Italy

${ }^{26}$ Medical Oncology Unit, Centro di Riferimento Oncologico di Aviano (CRO) IRCCS, Aviano, Italy

${ }^{27}$ Medical Oncology Unit, Ospedale Vito Fazzi, Lecce, Italy

${ }^{28}$ Oncologia Medica, Ospedale Villa Scassi, Genova, Italy

${ }^{29}$ UOC Oncologia di Belluno, Dipartimento di Oncologia Clinica, AULSS 1 Dolomiti, Ospedale S.Martino, Belluno, Italy

${ }^{30}$ Medical Oncology Division and Breast Unit, Senatore Antonio Perrino Hospital,

Brindisi, Italy

${ }^{31}$ Oncologia Medica, Ospedale della Versilia, Lido di Camaiore, Italy

${ }^{32}$ Medical Oncology, Ospedale Generale Regionale F Miulli, Acquaviva delle Fonti,

Puglia, Italy

${ }^{33}$ Medical Oncology, Camposampiero Hospital, ULSS 6 Euganea, Padova, Italy 
${ }^{34}$ Department of Oncology, Humanitas Clinical and Research Center, IRCCS, Milan, Italy

${ }^{35}$ Department of Biomedical Sciences, Humanitas University, Milan, Italy

${ }^{36}$ UC Oncologia Medica Uro-Ginecologica, Fondazione IRCCS Istituto Nazionale dei Tumori, Milano, Italy

${ }^{37}$ Federation of Italian Cooperative Oncology Groups (FICOG), Milano, Italy

${ }^{38}$ Medical Oncology Unit, AUSL-IRCCS of Reggio Emilia, Reggio Emilia, Italy

${ }^{39}$ Presidency of the Federation of Italian Cooperative Oncology Groups (FICOG), Milan, Italy

${ }^{40}$ Oncology, ASST Papa Giovanni XXIII, Bergamo, Italy

${ }^{41}$ Dipartimento di Discipline Chirurgiche, Oncologiche e Stomatologiche, Università degli Studi di Palermo, Palermo, Italy

${ }^{42}$ Medical Oncology, San Camillo Forlanini Hospital, Roma, Italy

${ }^{43}$ Department of Clinical and Molecular Medicine, Oncology Unit, Sant'Andrea Hospital, Sapienza University of Rome, Roma, Italy

Twitter Melissa Bersanelli @Bersa_Dr_Mel and Vincenzo Montesarchio @Vincenzo Montesarchio

Acknowledgements We acknowledge all the members of the Federation of Italian Cooperative Oncology Groups (FICOG), promoter and main sponsor of the INVIDla-2 study, in particular Evaristo Maiello, Roberto Labianca, and Luigi Bernardi. We acknowledge Roche S.p.A. (Gold sponsor), and Seqirus UK unlimited (Silver sponsor) for providing the funding for the INVIDla-2 study. We acknowledge the professionality of the FULLCRO staff for the outstanding management of the trial, in particular Claudia Guerrieri, Moira Cordisco and Elena Ottavianelli, and Data River for the eCRF validated platform. We acknowledge all the Investigators and Study Coordinators from the participating centers, for their outstanding collaborative efforts. Finally, we sincerely acknowledge our patients, their caregivers, and their general practitioner physicians for their trust in this study.

Collaborators Further Principal Investigators and Sub-Investigators to be acknowledged: Teresa Zielli, Fabiana Perrone, Chiara Casartelli, Agnese Cosenza, Leonarda Ferri, Roberta Minari, Salvatore lorio, Francesco Bonatti, Alessandro Leonetti, Paola Bordi, Fabiana Pratticò, Giuseppe Procopio, Diego Signorelli, Marina Chiara Garassino, Giovanni Schinzari, Lorenzo Calvetti, Rocco De Vivo, Umberto Basso, Alessio Cortellini, Fausto Barbieri, Roberto Sabbatini, Carlo Alberto Tondini, Camillo Porta, Maria Chiara Banzi, Giuseppe Fornarini, Sara Elena Rebuzzi, Nicola Battelli, Matteo Santoni, Claudia Mucciarini, Sergio Bracarda, Francesca Vignani, Gaetano Lacidogna, Andrea Bonetti, Lucia Longo, Donata Sartori, Editta Baldini, Michele Guida, Mauro lannopollo, Roberto Bordonaro, Debora Pezzuolo, Luigi Cavanna, Maria Francesca Morelli, Fausto Meriggi, Pierosandro Tagliaferri, Claudia Corbo, Angela Gernone, Davide Tassinari, Veronica Prati, Simona Carnio, Massimiliano Spada, Pasqualina Giordano, Angela Maria Dicorato, Francesco Carrozza, Claudio Verusio, Francesco Atzori, Stefania Gori, Anna Ceribelli, Antonino Castro, Rosa Rita Silva, Stefano Luzi Fedeli, Sara Pilotto, Michele Milella, Daniele Santini, Giordano Beretta, Franco Nolè, Vanja Vaccaro, Petros Giovanis, Elisabetta Garzoli, Elisa Franzini.

Contributors I declare that all authors contributed to the submitted work.

Funding This work was supported by Roche S.p.A. and Seqirus as Investigator Sponsored Research. No grant number is applicable. The study's funders had no role in study design, data collection, data analysis, data interpretation, or writing of the report.

Competing interests The Federation of Italian Cooperative Oncology Groups (FICOG) received funding for the present study by Seqirus and Roche S.p.A.; also received funding outside the present study by Astra Zeneca, Bristol-Myers Squibb (BMS), Sanofi.Melissa Bersanelli received funding for the present study by Seqirus and Roche S.p.A. (FICOG as Institution, no personal fees). She also received, outside the present work, research funding from Pfizer and Novartis (Institution); honoraria as a speaker at scientific events (personal fees) by Astra Zeneca, Bristol-Myers Squibb (BMS), Novartis and Pfizer; as consultant for advisory role (personal fees) by Novartis, BMS and Pfizer; for copyright transfer by Sciclone Pharmaceuticals. Ugo De Giorgi has served as a consultant for Astellas, Bayer, BMS, Ipsen, Janssen, Novartis, Pfizer, Sanofi and Pharmamar; he received research funding from AstraZeneca, Roche, and Sanofi; and received travel funds from BMS, Ipsen, Janssen, Pfizer, and Roche during the conduct of the study.Massimo Di Maio reports personal fees from Bristol Myers Squibb, personal fees from Merck Sharp MSD, Ipsen, Roche S.p.A., Eli-Lilly, AstraZeneca and Novartis; he also received research funding from Novartis.Vieri Scotti participated, with personal fees, to advisory boards and speaker's bureaus for Roche S.p.A. Saverio Cinieri declared international board for Eli Lilly international. Paolo Andrea Zucali acts in a consultant or advisory role for Sanofi, BMS, Pfizer, MSD, Astellas, Janssen, Ipsen, Novartis, all outside the scope of work. Marcello Tiseo received speakers' and consultants' fee from Astra-Zeneca, Pfizer, Eli-Lilly, BMS, Novartis, Roche, MSD, Boehringer Ingelheim, Otsuka, Takeda, Pierre Fabre, and institutional research grants from Astra-Zeneca, Boehringer Ingelheim. All remaining authors have declared no conflicts of interest.

\section{Patient consent for publication Not required.}

Ethics approval Local Institutional Review Board approval was required for each center for inclusion in the study. Written informed consent was obtained for all the enrolled patients. All the study procedures were in accordance with the precepts of Good Clinical Practice and the declaration of Helsinki.

Provenance and peer review Not commissioned; externally peer reviewed.

Data availability statement Data are available upon reasonable request. The study protocol and the validation certificate for the eCRF platform will be made available by the corresponding author upon request (bersamel@libero.it) at any time. Participants' data and the study dataset will not be made available until the end of the INVIDla-2 study, and in any case, not until the publication of final results, then they will be available by the corresponding author upon reasonable request.

Supplemental material This content has been supplied by the author(s). It has not been vetted by BMJ Publishing Group Limited (BMJ) and may not have been peer-reviewed. Any opinions or recommendations discussed are solely those of the author(s) and are not endorsed by BMJ. BMJ disclaims all liability and responsibility arising from any reliance placed on the content. Where the content includes any translated material, BMJ does not warrant the accuracy and reliability of the translations (including but not limited to local regulations, clinical guidelines, terminology, drug names and drug dosages), and is not responsible for any error and/or omissions arising from translation and adaptation or otherwise.

Open access This is an open access article distributed in accordance with the Creative Commons Attribution Non Commercial (CC BY-NC 4.0) license, which permits others to distribute, remix, adapt, build upon this work non-commercially, and license their derivative works on different terms, provided the original work is properly cited, appropriate credit is given, any changes made indicated, and the use is non-commercial. See http://creativecommons.org/licenses/by-nc/4.0/.

\section{ORCID iDs}

Melissa Bersanelli http://orcid.org/0000-0002-6527-6281

Vincenzo Montesarchio http://orcid.org/0000-0001-5545-8105

Sebastiano Buti http://orcid.org/0000-0003-0876-0226

\section{REFERENCES}

1 World Health Organization. Influenza fact sheets. Available: https:// www.who.int/en/news-room/fact-sheets/detail/influenza-(seasonal) [Accessed 2 Jan 2021].

2 luliano AD, Roguski KM, Chang $\mathrm{HH}$, et al. Estimates of global seasonal influenza-associated respiratory mortality: a modelling study. Lancet 2018;391:1285-300.

3 Casalegno J-S, Eibach D, Valette M, et al. Performance of influenza case definitions for influenza community surveillance: based on the French influenza surveillance network GROG, 2009-2014. Euro Surveill 2017;22:30504.

4 Commission implementing decision (EU) 2018/945 of June 22, 2018 on the communicable diseases and related special health issues to be covered by epidemiological surveillance as well as relevant case definitions. Official Journal of The European Union 2018;3:L170/24-25.

5 Cooksley CD, Avritscher EBC, Bekele BN, et al. Epidemiology and outcomes of serious influenza-related infections in the cancer population. Cancer2005;104:618-28.

6 Kunisaki KM, Janoff EN. Influenza in immunosuppressed populations: a review of infection frequency, morbidity, mortality, and vaccine responses. Lancet Infect Dis 2009;9:493-504.

7 Tinsley N, Zhou C, Tan G, et al. Cumulative antibiotic use significantly decreases efficacy of checkpoint inhibitors in patients with advanced cancer. Oncologist 2020;25:55-63.

8 Elkrief A, El Raichani L, Richard C, et al. Antibiotics are associated with decreased progression-free survival of advanced melanoma patients treated with immune checkpoint inhibitors. Oncoimmunology 2019;8:e1568812.

9 Rousseau B, Loulergue P, Mir O, et al. Immunogenicity and safety of the influenza A H1N1v 2009 vaccine in cancer patients treated 
with cytotoxic chemotherapy and/or targeted therapy: the VACANCE study. Ann Oncol 2012;23:450-7.

10 Bitterman R, Eliakim-Raz N, Vinograd I, et al. Influenza vaccines in immunosuppressed adults with cancer. Cochrane Database Syst Rev 2018;2:CD008983.

11 Pedrazzoli P, Baldanti F, Donatelli I, et al. Vaccination for seasonal influenza in patients with cancer: recommendations of the Italian Society of medical oncology (AIOM). Ann Oncol 2014;25:1243-7.

12 Poeppl W, Lagler H, Raderer M, et al. Influenza vaccination perception and coverage among patients with malignant disease. Vaccine 2015;33:1682-7.

13 Läubli H, Balmelli C, Kaufmann L, et al. Influenza vaccination of cancer patients during PD-1 blockade induces serological protection but may raise the risk for immune-related adverse events. $J$ Immunother Cancer 2018;6:40.

14 Weber JS, Hamid O, Chasalow SD, et al. Ipilimumab increases activated $\mathrm{T}$ cells and enhances humoral immunity in patients with advanced melanoma. J Immunother 2012;35:89-97.

15 Bersanelli M, Giannarelli D, Castrignanò P, et al. Influenza vaccine indication during therapy with immune checkpoint inhibitors: a transversal challenge. The INVIDla study. Immunotherapy 2018;10:1229-39.

16 Chong CR, Park VJ, Cohen B, et al. Safety of inactivated influenza vaccine in cancer patients receiving immune checkpoint inhibitors. Clin Infect Dis 2020;70:193-9.

17 Awadalla M, Golden DLA, Mahmood SS, et al. Influenza vaccination and myocarditis among patients receiving immune checkpoint inhibitors. J Immunother Cancer 2019;7:53.

18 Failing JJ, Ho TP, Yadav S, et al. Safety of influenza vaccine in patients with cancer receiving pembrolizumab. JCO Oncol Pract 2020;16:e573-80.

19 Rubin LG, Levin MJ, Ljungman P, et al. 2013 IDSA clinical practice guideline for vaccination of the immunocompromised host. Clin Infect Dis 2014;58:e44-100.

20 US Department of Health and Human Services. Common terminology criteria for adverse events (CTCAE) version 5.0; 2017.

21 World Health Organization. Regional officer for Europe. influenza vaccination coverage and effectiveness. Available: https://www. euro.who.int/en/health-topics/communicable-diseases/influenza/ vaccination/influenza-vaccination-coverage-and-effectiveness; [Accessed 2 Jan 2021].

22 Kang CK, Kim H-R, Song K-H, et al. Cell-Mediated immunogenicity of influenza vaccination in patients with cancer receiving immune checkpoint inhibitors. J Infect Dis 2020;222:1902-9.

23 Loulergue P, Alexandre J, lurisci I, et al. Low immunogenicity of seasonal trivalent influenza vaccine among patients receiving docetaxel for a solid tumour: results of a prospective pilot study. $\mathrm{Br} J$ Cancer 2011;104:1670-4.

24 Bayle A, Khettab M, Lucibello F, et al. Immunogenicity and safety of influenza vaccination in cancer patients receiving checkpoint inhibitors targeting PD-1 or PD-L1. Ann Oncol 2020;31:959-61.

25 Keam B, Kang CK, Jun KI, et al. Immunogenicity of influenza vaccination in patients with cancer receiving immune checkpoint inhibitors. Clin Infect Dis 2020;71:422-5.

26 AIOM Ricerca Studi. Sotto-studio ancillare INVIDla-bios: caratterizzazione immuno-biologica su sangue periferico in pazienti oncologici arruolati nello studio osservazionale INVIDla-2. Available: https://studiclinici.aiom.it/studi-clinici/ricerca-studiclinici/sotto-studio-ancillare-invidia-bios-caratterizzazioneimmuno-biologica-su-sangue-periferico-in-pazienti-oncologiciarruolati-nello-studio-osservaziona/6-511-13160 [Accessed $10 \mathrm{Jan}$ 2021].

27 Kalil AC, Thomas PG. Influenza virus-related critical illness: pathophysiology and epidemiology. Crit Care 2019;23:258.

28 Suryaprasad A, Redd JT, Hancock K, et al. Severe acute respiratory infections caused by 2009 pandemic influenza A (H1N1) among American Indians-southwestern United States, May 1-July 21, 2009. Influenza Other Respi Viruses 2013;7:1361-9.

29 Bersanelli M, Giannarelli D, De Giorgi U, et al. Symptomatic COVID-19 in advanced-cancer patients treated with immune-checkpoint inhibitors: prospective analysis from a multicentre observational trial by FICOG. Ther Adv Med Oncol 2020;12:1758835920968463.

30 Solomon DA. Seasonal influenza vaccination. JAMA 2020;324:1362.

31 Solomon DA, Sherman AC, Kanjilal S. Influenza in the COVID-19 era JAMA 2020;324:1342-3. 